\title{
Intracerebral giant hydatidosis in an adult woman - A case report
}

\author{
Prakash Mahantshetti ${ }^{1}$, Ravi Ichalakaranji ${ }^{2, *}$, Suhasini $\mathbf{H}^{3}$ \\ ${ }^{1}$ Assistant Professor, ${ }^{2,3}$ Resident, ${ }^{1,2}$ Dept. of Neurosurgery, ${ }^{3}$ Dept. of ENT, Jawaharlal Nehru Medical College, Belgaum, \\ Karnataka, India
}

*Corresponding Author:

Email: ravi.ichalkaranji@gmail.com

\begin{abstract}
Intracranial hydatid cysts account for $0.5-3 \%$ of all the cases of hydatid disease and contribute to $1-2 \%$ of all the intracranial space occupying lesions. Rare cases of intracranial hydatid disease have been reported in India. In India, the hydatid disease is more commonly seen in the Kurnool district of Andhra Pradesh, Madurai district of Tamil Nadu and in Punjab.

Here we present a case of a 35-year-old female patient presented to us with diminution of vision, diplopia and headache since 10 days. Her neurological assessment showed higher mental functions within normal limit and Visual acuity - 6/12 right eye and 6/9 left eye, with normal fundus \& right $6^{\text {th }}$ cranial nerve palsy. MRI brain $(\mathrm{P}+\mathrm{C})$ done was suggestive of intra-axial well defined cystic lesion in right tempero- frontal lobe which is T1 hypointense and T2 hyperintense measuring $4.5 \times 5.25 \mathrm{cms}$ Patient was subjected for surgical excision of cyst. Post operatively patient improved well with no neurological deficits. Histopathology of the lesion confirmed hydatid cyst. Patient was put on oral albendazole $10 \mathrm{mg} / \mathrm{kg}$ per day and continued for 12 weeks. Patient is on regular follow up with us, with no neurological deficits.
\end{abstract}

Keywords: Intracerebral hydatidosis, Echinococcus, Dowling-Orlando technique, Albendazole.

\section{Introduction}

Intracranial hydatid cysts account for $0.5-3 \%$ of all the cases of hydatid disease and contribute to $1-2 \%$ of all the intracranial space occupying lesions. ${ }^{1}$ Hydatid disease is caused by the infestation of the larvae of echinococcus (platyhelminth). The definitive host are various carnivores, the common being the dog and man is accidental host infested by feco-oral route. Hydatid cysts constitute only a small percentage of space occupying lesions of the brain. Rare cases of intracranial hydatid disease have been reported in India including the study done by Ramamurthi et al $(0.2 \%)$ and Raja reddy et al $(0.2 \%)$ Hydatid disease is endemic in the middle east, Mediterranean countries, South America, North Africa and Australia. ${ }^{1}$ In India, the hydatid disease is more commonly seen in the Kurnool district of Andhra Pradesh, Madurai district of Tamil Nadu and in Punjab. ${ }^{2}$

\section{Case Report}

A 35-year-old female patient presented to us with diminution of vision, diplopia and headache since 10 days. She had no h/o seizures, vomiting, focal deficits, ataxia, hemiparesis and altered consciousness.

On examination: Patient was conscious, oriented.

Neurological assessment: Higher mental functions were within normal limit.

Visual acuity: 6/12 right eye and 6/9 left eye, With fundus: Normal

Cranial nerve examination showed Right $6^{\text {th }}$ nerve palsy and other cranial nerves within normal limit.

Diplopia charting showed Uncrossed diplopia in primary supraduction, abduction, dextroelevation.

MRI brain $(\mathrm{P}+\mathrm{C})$ done was suggestive of intra-axial well defined cystic lesion in right Tempero - frontal lobe which is $\mathrm{T} 1$ hypointense and $\mathrm{T} 2$ hyperintense measuring $4.5 \times 5.25 \mathrm{cms}$.

Patient was subjected for surgical excision of cyst. Intraoperatively, after fronto temporal craniotomy and cortisectomy there was accidental rupture of the cyst wall and extravasation of cystic fluid. Subsequently, multiple nodular structured tiny cysts with fluid was found in the cavity which was suspected to be hydatid cyst following which the fluid with the contents was carefully suctioned without spillage and sent for histopathological examination. After which, the cavity was irrigated with $3 \% \mathrm{NaCl}$ and closure was done. Ultrasound abdomen and chest $\mathrm{X}$ ray was done which revealed no evidence of cystic lesion. Post operatively patient improved with no neurological deficits. Patient was put on oral albendazole $10 \mathrm{mg} / \mathrm{kg}$ per day and continued for 12 weeks.

Histopathology of the lesion showed presence of cystic parasitic structure with lamellated ectocyst and focal germinal cell layer. Few invaginated scolices are seen showing hooklets and suckers.
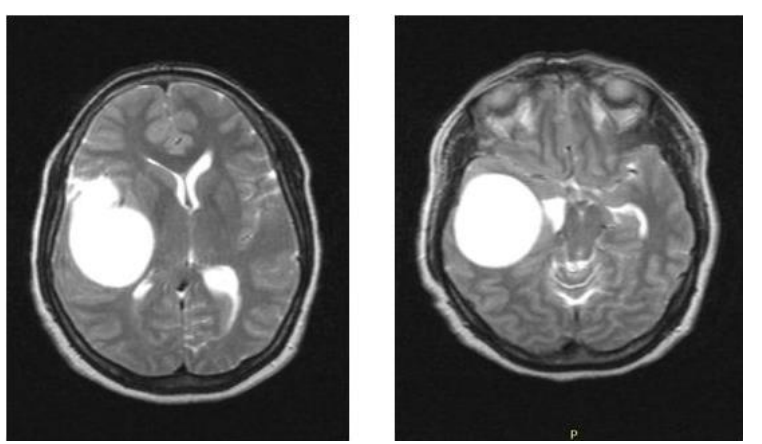

Fig. 1: T2 MRI brain showing cystic lesion in right frontotemporal lobe 

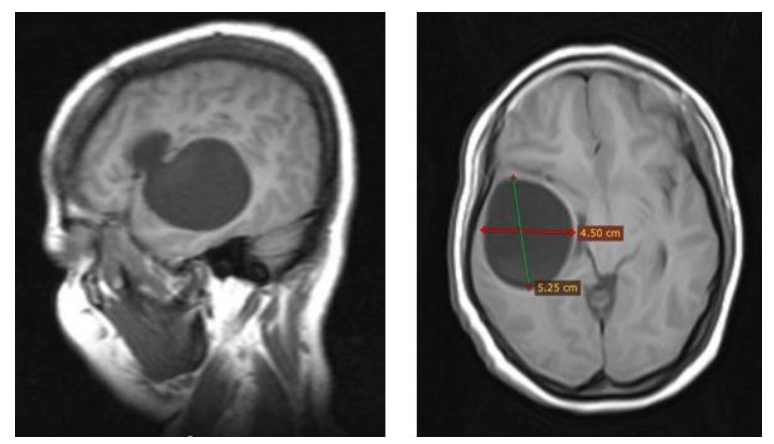

Fig. 2: MRI brain T1 showing cystic lesion in right frontotemporal lobe

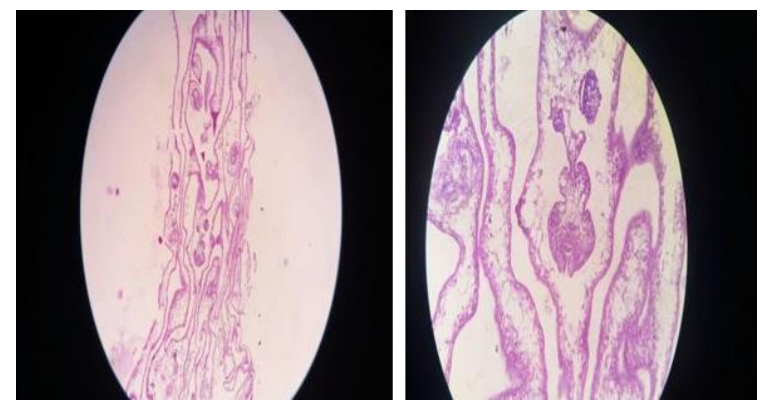

Fig. 3: HPR showing cystic parasitic structure with lamellated ectocyst and focal germinal cell layer

\section{Discussion}

Hydatid disease is a rare disease caused by the larval stage of the cestode Echinococcus granulosus. Dogs are definitive host and man is intermediate host. The eggs hatch inside the intestines and the oncospheres penetrate its walls, they reach the portal circulation and then reach the liver where they can cause cystic lesions. They can move to reach the lung. After those two filters some could still make it to the systemic circulation and could reach the brain. There are two main forms of echinococcosis: cystic hydatid disease (caused by Echinococcus granulosus) and alveolar hydatid disease (caused by Echinococcus multilocularis). ${ }^{3}$

Intracranial hydatid disease is rare; with reported incidence of $1-2 \%$ of all cases with hydatid disease. ${ }^{4}$

Intracranial hydatid cysts are more frequently located in the supratentorial compartment. The parietal lobe is the commonest site. The other less common sites reported are skull, cavernous sinus, eye ball, pons, skull, extradural, cerebellum and ventricles. ${ }^{5}$

Brain hydatid cysts can be primary or secondary. Primary hydatid cysts occur as a result of direct invasion of larva that managed and filtered via liver and lung to the brain and they are usually solitary. Secondary hydatid cysts occur as a result of rupture of primary cysts in others organs then reaching by embolization to the brain and they are usually multiple and infertile. ${ }^{6}$ The primary cysts are fertile as they contain scolices and brood capsules, hence rupture of primary cyst can result in recurrence. The secondary multiple cysts results from spontaneous, traumatic or surgical rupture of the primary intracranial hydatid cyst and they lack brood capsule and scolices. The secondary intracranial hydatid cysts are therefore, infertile and the resultant risk of recurrence after their rupture is negligible. Cysts develop insidiously, usually being asymptomatic initially. Cystic hydatid disease results in seizures or increased intracranial pressure of subacute onset and has a progressive course, often in association with focal neurological deficits. ${ }^{7}$ Orbital involvement in patients with cystic hydatid disease is manifested as proptosis and ophthalmoplegia. The neurological manifestations progress more rapidly and are more severe with alveolar hydatid disease than with cystic hydatid disease.

Diagnosis of cerebral hydatid disease is based mainly on neuroimaging and could be supported with serological tests (ELISA have a sensitivity of $85 \%$ ) and confirmed only with histopathological studies. ${ }^{8}$

MRI is considered superior to CT in the diagnosis and in preoperative assessment. MR imaging of Neurohydatidosis reveals a well-defined, circumscribed, solitary, spherical, intra-axial cystic lesion. Cysts lie in the territory of the middle cerebral artery commonly in parietal lobe. They show no enhancement with Gadolinium, no calcification and typically no surrounding oedema. They show no communication to the ventricles or to subarachnoid space. They can cause mass effect, midline shift and hydrocephalus. ${ }^{9,11}$ The definitive management of a cerebral hydatid cyst is surgical removal of the entire cyst with utmost care to avoid its rupture. The Preferred method is by using Dowling-Orlando technique. The complications associated with the Dowling technique include subdural effusions and intracranial hemorrhages. To avoid these complications, some surgeons puncture the cyst, aspirate its contents, irrigate the cyst with a hypertonic saline solution, and then remove the shrunken cyst. ${ }^{12}$ Alveolar hydatid disease is invasive, and total surgical removal usually requires resection of adjacent tissue. Medical treatment using Albendazole alone or in combination with praziquintal may be administered post-operatively for 3-6 months. Albendazole may be used in patients who are not candidates for surgical resection of lesions, as prophylactic therapy for those at risk for accidental rupture of the cysts preoperatively, or to treat recurrent cystic hydatid disease after surgery. ${ }^{12}$

\section{Conclusion}

Intracerebral hydatidosis is a rare entity seen in adults and should be considered as one of the differential diagnosis of intracranial cysts. USG abdomen and chest $\mathrm{x}$-ray should be the part of surgical work up. MRI brain always remains the gold standard for assessing these lesions. Serological tests are supportive but not specific. Surgical excision should be planned meticulously and oral Albendazole postoperatively will be the most effective regimen. 


\section{References}

1. Ismail Ibrahim Ismail, Sarah Salama Foad: Primary Cerebral Hydatid Cyst in an Adult Female Presenting with Seizures and Hemiparesis: A Case Report J Neurol Disord 2015,3:2.

2. Dharker SR: Hydatid disease. In: Text Book of Neurosurgery, Second edition. Eds. Ramamurthi B, Tandon PN. Churchill Livingstone, New Delhi 1996;53544.

3. G Jacquet, J Godard, A Czorny: [Cerebral alveolar echinococcosis. Apropos of a surgically-treated case. Review of the literature]. Neurochirurgie 1992;38:36267.

4. Erashin Y, Mutluer S, GuzelbagE: Intracranial hydatid cysts in children. Neurosurg 1993;33:219-24.

5. Gupta S, Desai K, Goel A: Intracranial hydatidcyst: a report of five cases and review of literature.

6. Polat P, Kantarci M, Alper F (2003) Hydatid disease from head to toe. Radiographics 23:475-94.

7. DM Patrikar, KR Mitra, VR Bhutada: Cerebral hydatid di sease. Autralas Radiol 1993;37:226-27.

8. Binesh F, Mehrabanian M, Navabii H (2011) Primary brain hydatosis. BMJ Case Rep.

9. Tuzun M and Hekimoðlu B (1998) Hydatid disease of the CNS. Imaging features. AJR 171:1497-500.

10. El-Shamam O, Amer T, El-Atta MA (2004) Magnetic resonance imaging of simple and infected hydatid cysts of the brain. Magn Reson Imaging 22:1339-40.

11. Ravalji M, Kumar S, Shah AK, Vaghela DU, Makwana GJ, et al. (2006) CT and MRI features of the typical and atypical intracranial hydatid cysts: Report of five cases. Ind J Radiol Imaging 16:727-32.

12. Oscar H. Del Brutto, Juan J. Figueroa, Hector H. Garcia parasaitic infections. Youmans and Winn Neurological Surgery. Seventh edition 2017. 